\title{
Implication of viral microRNAs in the genesis and diagnosis of Epstein-Barr virus-associated tumors (Review)
}

\author{
XIANGNING ZHANG ${ }^{1}$, YUDONG YE ${ }^{2}$, MING FU $^{2}$, BIYING ZHENG $^{3}$, QIANHUI QIU ${ }^{4}$ and ZUNNAN HUANG ${ }^{1}$ \\ ${ }^{1}$ Department of Pathophysiology, Chinese-American Collaborative Cancer Institute, Guangdong Provincial \\ Key Laboratory of Medical Molecular Diagnostics, Guangdong Medical University, Dongguan, Guangdong 523808; \\ ${ }^{2}$ Department of Otolaryngology, Dongguan City People's Hospital, Dongguan, Guangdong 523059; ${ }^{3}$ Department of \\ Clinical Microbiology, College of Laboratory Medicine, Guangdong Medical University, \\ Dongguan, Guangdong 523808; ${ }^{4}$ Department of Otolaryngology, Pearl River Hospital, \\ Southern Medical University, Guangzhou, Guangdong 510280, P.R. China
}

Received September 14, 2018; Accepted June 26, 2019

DOI: $10.3892 / 01.2019 .10713$

\begin{abstract}
The Epstein-Barr virus (EBV) is tightly associated with a variety of human tumors, including Burkitt lymphoma and acquired immune deficiency syndrome-related lymphoma of B-cell origin, as well as nasopharyngeal carcinoma and gastric cancer of epithelial origin. The virus latently infects the host cells and expresses proteins and non-coding RNAs to achieve malignancy. MicroRNAs (miRNAs or miRs) are small RNAs consisting of 19-25 nucleotides, which directly bind to the 3'-untranslated region of mRNAs to promote degradation and inhibit translation of mRNAs. EBV-encoded miRs are generated from two regions of the viral genome, within the apoptosis regulator BHRF1 gene locus and near the BamHI A region in a latency type-dependent manner. In addition, EBV-encoded miRs epigenetically regulate the expression of molecules that are effectors of the cell cycle progression, migration, apoptosis and innate immunity, serving a vital role in supporting viral replication and occurrence of EBV-associated tumors. The feasibility of using such miRs as biomarkers for the diagnosis and prognosis of EBV-associated tumors is currently under investigation.
\end{abstract}

Correspondence to: Dr Xiangning Zhang, Department of Pathophysiology, Chinese-American Collaborative Cancer Institute, Guangdong Provincial Key Laboratory of Medical Molecular Diagnostics, Guangdong Medical University, 1 Xincheng Avenue, Songshan Lake Scientific, Technology and Industrial Park, Dongguan, Guangdong 523808, P.R. China

E-mail: zhangxn_2006@126.com

Abbreviations: miR, microRNA; EBV, Epstein-Barr virus; EBNA, EBV-determined nuclear antigen; LMP, latent membrane protein; NPC, nasopharyngeal carcinoma; EBVaGC, EBV-associated gastric cancer; PTLD, post-transplantation lymphoproliferative disorder; BHRF1, Bam $\mathrm{HI}$ reading frame 1; BART, BamHI A region transcripts

Key words: Epstein-Barr virus, microRNA, nasopharyngeal carcinoma, gastric carcinoma, tumorigenicity, immune regulation

\section{Contents}

1. Introduction

2. Cell type-dependent expression spectrum of EBV-encoded miRs

3. EBV-encoded miRs target cellular genes to regulate the biological activities of the host

4. EBV-encoded miRs as biomarkers for the diagnosis and prognosis of EBV-associated tumors

5. Conclusion

\section{Introduction}

The Epstein-Barr virus (EBV) is a ubiquitous lymphotropic herpesvirus within the human gamma herpesvirus group. This virus infects $>95 \%$ of individuals in a population during childhood and early adolescence in an asymptomatic process. In adolescent individuals, it may cause infectious mononucleosis, which manifests as a fever, pharyngitis and lymphadenopathy $(1,2)$.

EBV establishes its infection mainly in two types of cells, lymphocytes and epithelial cells, and this infection persists in the host, establishing latency. EBV infection is associated with the occurrence of numerous diseases, including: Nasopharyngeal carcinoma (NPC) and EBV-associated gastric cancer (EBVaGC) of epithelial origin; Burkitt lymphoma (BL), acquired immune deficiency syndrome-related lymphoma (ARL) and post-transplantation lymphoproliferative disorder (PTLD) of B-cell origin; natural killer (NK)/T-cell lymphoma of T-cell origin; lymphoid tumors; and Hodgkin's disease. The viruses that replicate in cells with genetic lesions may contribute to oncogenesis. Previous data suggested that EBV infection is responsible for the clonal expansion of premalignant nasopharyngeal epithelial cells (3).

In EBV-associated lymphomas, the virus adopts different types of latent infection, ranging from type I-restricted expression patterns of the viral genome to a wider spectrum of type II and type III, under which the full spectrum of viral genomic products is expressed. Lymphomas with latency type I EBV 
infection, which is exemplified by BL, certain ARLs and PTLD, carry cytogenetic or genetic mutations leading to oncogene activation or inactivation of tumor suppressor genes (4). In diffuse large B-cell lymphoma (DLBCL), B-cell lymphoma 6 (BCL-6) is frequently translocated, while other genetic alterations are also present, including cMyc and BCL-2 translocations, as well as p53, N-RAS and tumor necrosis factor (TNF)- $\alpha$-induced protein 3 mutations (4). All cases of BL possess chromosomal translocation resulting in Myc immunoglobulin gene juxtaposition, promoting the transformation potential of the Myc oncogene.

In lymphomas with latency type III EBV infection, transforming viral proteins are expressed. Activation of the Notch-1 signaling pathway by EBV-determined nuclear antigen 2 (EBNA-2) is responsible for the transformation of this type of lymphoma (5). The membrane integral proteins, latent membrane protein 1 (LMP1), LMP2A and LMP2B, possess transforming potential. Among these, LMP1 is the viral homolog of mammalian cluster of differentiation 40, which is present as an active TNF receptor. LMP1 aggregates on the cell surface, and integrates signals of growth and proliferation by activating the nuclear factor (NF)- $\kappa \mathrm{B}$ and c-Jun amino-terminal kinase signaling pathways when the plasmic carboxyl-terminal tail of LMP1 associates co-factors such as TNF receptor-associated factors (TRAFs) and TNF receptor type 1-associated DEATH domain protein (6). By contrast, LMP2A, which is also expressed in human tumors with latency II and III EBV infections, is homologous to the mammalian B-cell receptor. It also contributes to NF- $\kappa \mathrm{B}$ signaling by controlling TRAF2 expression, which in turn is important for LMP1 signaling (7).

MicroRNAs (miRNAs or miRs) are a group of newly emerging non-coding small RNAs that bind to the 3'-untranslated region (3'-UTR) of mRNAs of specific genes and inhibit the translation of proteins. A study examining the EBV-infected GC cell line AGS revealed that miRs encoded by EBV bind to the 3'-UTRs of transcripts of max-interacting protein 1, activating transcription factor 5 (ATF-5) and ATF-6 (8). By abrogating the inhibition of Myc, EBV encoded BART miRs contribute to the activation of proto-oncogene Myc. As members of the unfolded protein group, ATF-5, ATF- 6 and X-box-binding protein 1, induced by BART miRs, subsequently inhibit the expression of the EBV-encoded lytic protein transactivator protein BZLF1 to maintain the latency of the EBV infection in host cells. The decreased expression of ATF-5 and ATF-6 facilitates entry into the viral lytic cycle (8). These findings suggested that, in addition to coding for protein products, EBV also generates non-coding RNAs to contribute in host-virus interaction to mediate malignant transformation.

miRs consist of 19-25 nucleotides (nt) in length and are generated from mRNA catalyzed by type II DNA polymerases, which initially forms primary RNA and then matures into miRNA. It is known that miRs possess the ability to form a stem-loop, imperfectly complementary, hairpin structure with a 33-base pair (bp) stem and a terminal loop on mRNAs (9).

Drosha, a type III RNAse enzyme, initially forms a microprocessor complex; next, together with helicases, it recognizes and cleaves the folded miRs located 11 bp away from the junction between the single- and double-stranded RNA at the base of the hairpin stem $(10,11)$. A pre-miRNA that is $60-100 \mathrm{nt}$ long is then generated and retains the hairpin of its precursor. Furthermore, in the cytoplasm, the RNA-induced signaling complex containing Dicer (an RNAse type III enzyme) cleaves off the terminal loop from the pre-miRNA to generate a mature double-stranded $\mathrm{miR}(10,11)$.

In addition to targeting the 3'-UTRs of mRNAs of specific genes, the binding sites of miRs in other regions on mRNA strands have been described (9). miRs accelerate the degradation of mRNAs, as well as to inhibit the translation of mRNAs. It has also been reported that the expression of miRs affects multiple biological processes in the human body, as well as viral parasitism and replication.

The present review aims to summarize the latest advances in the study of EBV encoded miRs in regards with their biogenesis, cell type dependent expression profile, activities in regulating host immunity, apoptosis, cell cycle and tumor progression, to explore their possible application in diagnosis and prognosis of EBV associated human tumors.

\section{Cell type-dependent expression spectrum of EBV-encoded miRs}

miRs are a group of non-coding small RNAs that do not encode any protein products. In EBV-infected cells, the viral genome encodes multiple proteins and miRs to maintain the malignant phenotype.

Five viral miRNAs were first identified in 2004 in EBV-infected cells [13]; over the last 10 years, additional EBV miRNAs were identified by sequencing studies [15-17]. At least 44 mature miRNAs arise from the $25 \mathrm{EBV}$ precursor miRNAs (pre-miRNAs): Three miRNAs flank the BHRF1 ORF encoding a viral Bcl2 homolog, including miR-BHRF1-1, miR-BHRF1-2, miR-BHRF1-3, and two large clusters of miRNAs arise from introns within the BART region. The three BHRF1 miRs are expressed in EBV latency III infections (12-14). miR-BHRF1-1 is located at the 5 ' end of the BHRF1 lytic mRNA transcription start site and overlaps the TATA box of the EBV replication-activated BHRF1 promoter, whereas miR-BHRF1-2 and miR-BHRF1-3 are located in the 3'-UTR of the BHRF1 (13).

Among 25 miRs encoded by the EBV genome, three miRs arise in the BHRF 1 locus, and the remaining 22 miR-BARTs are located in three clusters $(15,16)$. The BART miRs are divided into two subclusters: Subcluster 1 , which includes miR-BART1, 3-6 and 15-17, and subcluster 2 that consists of miR-BART7-14 and 18-21 (17). As indicated in Fig. 1, subtypes of the BART cluster 2 members, including miR-BART18-3p, 7-5p, 10-3p, 10-5p, 11-3p, 13-5p and 14-5p, were expressed in latency type III infections $(18,19)$. In addition, miR-BART2-5p and miR-BART2-3p have been reported to be the downstream members of these two subclusters (20). The EBV-encoded BART miRs are expressed in virtually all EBV infection-associated human tumors, ranging from BL (latency I), NK/T-cell lymphoma (latency I) and EBV aGC (latency I), to Hodgkin disease (latency II), NPC (latency II), and EBV-associated B lymphoma and PTLD (latency III).

Expression of EBV-encoded miRs in B-cell lymphomas. Despite the full transformation potential in B lymphocytes, the prototype EBV strain B95-8 contains a deletion in the 


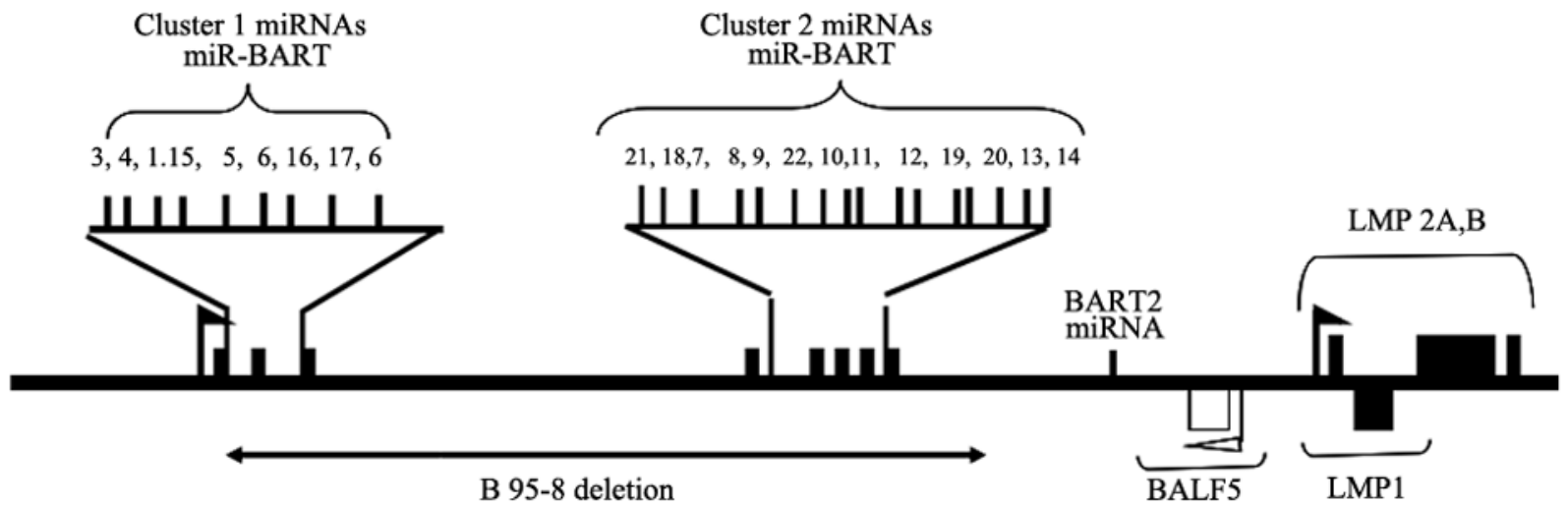

Figure 1. Location of EBV-encoded BART miRs on the viral genome. The miRs are grouped into clusters 1 and 2; the profile and molecular target of individual miRs have been reported. Promoters and genes (pennant and box, respectively) in EBV-positive nasopharyngeal carcinoma tumors (latency II) are shown. Half of the miR-BARTs in cluster 1 and all of the miR-BARTs in cluster 2 are deleted in the B95-8 strain [adapted from the studies by Lo et al (19) and Fernandes et al (55)]. EBV, Epstein-Barr virus; miR, microRNA; BART, BamHI A region transcripts.

BART region, which encodes part of the BART clusters of miRs (20). When B cells were immortalized to form the LCL by infecting them with the B95-8 strain of EBV, the miRs from the affected region were not expressed (21). BHRF1 miRNAs are only found in cells that harbor the virus in type III latency, such as PTLD arising during immunosuppression (22), but not for instance in GC or NPC (17,23-25).

Expression profile of EBV-encoded miRs in NK/T cells. An analysis of the EBV antigenic expression profile of EBV-positive NK/T-cell lymphomas arising in the nasal cavity of immunocompetent patients revealed that EBV adopts type I latency, as LMP1 or EBNA2 are not expressed (26). In addition, miRs derived from the BHRF1 cluster were not found in the tumor cells. In NPC, the EBV miRs accounted for 5-19\% of the total miRs, whereas the viral miRNAs in NK/T-cell lymphoma represented only $2.3 \%$ of the total miRs. The five most expressed EBV-miRs, BART7, 5, 11-5p, 1-5p and 19-3p, accounted for $\sim 50 \%$ of the viral-encoded miRs and $\sim 1 \%$ of the total miRs $(15,26)$. Similar to the expression profile of EBV-encoded miRs in NPC tumor biopsies, all the analyzed BART miRs were detected in peripheral T-cell lymphoma samples $(26,27)$.

Expression profile of EBV-encoded miRs in gastric cancer. Non-invasive gastric carcinoma tissues are associated with latent infections of EBV (28). In EBVaGC, the tumor cells exhibitEBV latency I infections and express the EBV-encoded small RNA EBNA1. In certain human GC cells, LMP2A could also be detected, whereas LMP1 is often absent (29). As type I latency cells, EBVaGC cells express different BART miRs. A comprehensive profiling of EBV-miRNAs was analyzed in patients with gastric cancer (17). The prevalence of EBVa GC was 5.0\% (52 out of 1039) in our series. The most abundant EBV-miRNAs of EBVa GC were Bart4, followed by Bart11, Bart2, Bart6, Bart9, and Bart18, in the decreasing order. Of them, Bart9 exhibited the same seed sequence as to hsa miR-200a and miR-141. Expression of E-cadherin of EBV-positive SNU-719 was increased after BART9 knockdown. In infected cells, the viral BART miRs were expressed at high levels, and a small fraction of cellular miRs exhibited consistently decreased expression with specific downregulation of tumor suppressor miRs. Furthermore, the AGS-EBV cell lines was reported to have an expression pattern similar to that of the NPC cells and other EBV-associated epithelial malignancies in which the BART miRs are highly expressed and the BHRF1 miRs are barely detectable (17).

Expression profile of EBV-encoded miRs in NPC. Two methods have been commonly used to determine the abundance of BART miRs in NPC tissues and cell lines. One of these methods is stem-loop polymerase chain reaction (PCR) for performing quantitative (q)PCR with very small RNA templates, in which miRs serve as such templates. Profiling the expression levels of BART miRs from the EBV-harboring NPC cell line C666-1 and NPC biopsies was performed using this method $(14,24,25,30)$. Furthermore, relative abundance of different BART miRs can also be determined by direct sequencing of miR species into small RNA libraries. Several BART miRs were detected in terms of relative abundance in C666-1 cells, including miR-BART15, 10 and 19-3p. The relative abundance of the miRs varies considerably when detected with these two different methods, namely qPCR and direct sequencing $(14,25,31,32)$.

In a previous study, the profiling of 39 of the 40 known mature EBV miRs was assayed with a multiplex reverse transcription-PCR (33). With this approach, a comprehensive profile of EBV miRs in primary NPC tumors, including estimates of miRNA copy number per tumor cell, was obtained. It was reported that BART-derived miRs are present in a wide range of copy numbers from $<10^{3}$ per cell in both primary tumors and the widely used NPC-derived C666-1 cell line (33).

miR-BART7 is an miRNA that is encoded by cluster 2 in the EBV genome and is highly expressed in undifferentiated NPC-derived cells infected with EBV (11,18). Mature EBV-miR-BART7 (MIMAT0003416) is a single-stranded molecule containing 22 nt (5'-caucauaguccaguguccaggg-3'). Further investigation confirmed that EBV-miR-BART7 is also highly expressed in NPC biopsies, suggesting that it may have an oncogenic role (33). 


\section{EBV-encoded miRs target cellular genes to regulate the biological activities of the host}

As discussed earlier,EBV-encoded miRs are expressed in a cell type-dependent manner. The BHRF1 3'-UTR in the BHRF1 cluster with miRs 1-1, 1-2, 1-3 and the third immediately 5 ' to the BHRF1 lytic mRNA transcription start site (34) are mainly expressed by the EBV strain harbored in latency type III cells, including EBV-transformed cells in vitro, PTLD and B-cell lymphomas in complication with PTLD. BART miRs are expressed in latency types I and II, adopted by EBV-positive cell lines including BL and NPC, respectively (24).

In a previous study, a virus mutant D123 that lacks all three members of cluster BHRF1 miRs was constructed (35). The B-cell transforming capacity of the D123 EBV mutant was decreased by $>20$-fold relative to the wild-type or mutant viruses. However, the B cells harboring EBV deletion with BHRF1 miRs displayed higher latent gene expression levels and latent protein production when compared with their wild-type counterparts. The growth of B cells infected with the deletion-mutant virus was markedly decreased, and the percentage of cells entering the $S$ phase of the cell cycle was two-fold reduced. Therefore, BHRF1 miRs accelerate B-cell expansion at lower latent gene expression levels. The data of this previous study suggested that expressed miRs expand the viral reservoir and reduce the viral antigenic load, hence facilitating the persistence of EBV in the host (36). EBV-encoded miRs also contribute to carcinogenesis by apoptosis inhibition and immune evasion.

EBV has been reported to be tightly associated with NPC (36-38). The virus adopts the latency II characteristic of restricted viral genomic expression profiles due to the immune competence of the hosts. The expression of viral proteins has been demonstrated to be downregulated, whereas EBV-encoded miRs in the BART cluster were highly expressed $(14,19)$. EBV-miR-BART5-3p was reported to be upregulated and to promote the growth of NPC and gastric cancer cells. BART5-3p directly binds to the 3'-UTR of mRNA of the p53 coding gene, and downregulates cyclin dependent kinase inhibitor $1 \mathrm{~A}$ (CDKN1A), BAX and FAS expression, which has been reported to lead to acceleration of the cell cycle progression and inhibition of cell apoptosis. It has also been observed that BART5-3p facilitated the degradation of p53 $(37,39)$. The findings suggest a mechanism underlying the strategies utilized by EBV to maintain latent infection and promote the development of carcinomas associated with EBV infection (36).

The targets of EBV-encoded multiple cancer-related proteins have been described. These target molecules include BCL-2-interacting mediator of cell death (Bim) (40), p53 upregulated modulator of apoptosis (PUMA) (41), determination of interleukin- 4 commitment 1(DICE1) (42), the tumor suppressor phosphatase and tensin homolog (PTEN) $(43,44)$ and E-cadherin (45). BART miRs also target a number of genes associated with host immune regulation, including importin $7(43,46)$, Dicer $(44,47)$ and major histocompatibility complex class I-related chain B (48), as well as EBV encoded transforming proteins, LMP1 and LMP2 (49,50), as listed in Table I.

Impact of EBV-encoded miRs on the anti-EBV immune response. Viruses have evolved numerous strategies to evade host immune responses to support viral replication (51). It has been reported that growth of cells infected with mutant EBV with miR deletions is markedly slowed down (36). Evidence has suggested that EBV-encoded miRs promote the proliferation and survival of infected B cells and tumor cells, modulate immune evasion and reduce immune recognition against EBV (52-56).

It was demonstrated that the pre-miR-BHRF1-2 and 1-3 stem-loops are present in the 3'-UTR of transcripts encoding EBNA-leader protein (EBNA-LP; also known as EBNA5), an EBV antigen expressed early in viral infections (57). Excision of pre-miR-BHRF1-2 and 1-3 by Drosha to generate mature miRs destabilizes these mRNAs and reduces the expression of the encoded protein. Experiments using mutant EBV strains with inactivated pre-miR-BHRF1-2 and 1-3 revealed the upregulated expression of EBNA-LP, and EBNA-LP-regulated miRs and proteins, including LMP1 (49). The data suggested that the expression of BHRF1 miRs serves a role in immune evasion by reducing the levels of viral proteins that evoke immune response (56).

miRs have been known to modulate innate and adaptive immunity through transcriptionally regulating multiple genes. miRs encoded by EBV and other human herpesviruses are less immunogenic due to their small size; these miRs regulate the expression of both viral and host proteins $(52,55)$, and work efficiently to evade the anti-viral immune defense. It has been reported that herpes virus-encoded miRs affect host immunity by targeting the expression of ligands of NK cell receptors (48), chemokines (58) and inflammasome components $(59,60)$, and components of innate immune signaling pathways (61-64).

In EBV-transformed B cells and GC cells, miR-BART16 directly targets and downregulates CREB-binding protein, which is a key transcriptional coactivator in interferon (IFN) signaling (65). It also abrogates the production of IFN responsive genes in response to IFN- $\alpha$ stimulation and inhibits the antiproliferative effect of IFN- $\alpha$ in latently infected BL cells. By obstructing the type I IFN-induced antiviral response, miR-BART16 facilitates the establishment of a latent EBV infection and promotes viral replication (65).

Signaling of interleukin-1 (IL-1) serves an important role in inflammation and early activation of the antiviral innate immune response. EBV-encoded miRs have been identified to target the 3'-UTR of IL-1 receptor 1 mRNA, as confirmed by 3'-UTR luciferase reporter assays (66). This viral miR activity disrupts IL-1 autocrine and paracrine signaling loops and provides a survival advantage by dampening excessive inflammation that is detrimental to the infected cell.

Impact of EBV-encoded miRs on host cell apoptosis. As a form of programmed cell death, apoptosis is a host mechanism to eliminate unwanted cells in order to maintain homeostasis. It also functions to combat viral infection by triggering the death of virus-infected cells. Apoptosis is initiated and executed in complicated cascades (67); the apoptotic pathways in which mitochondria and caspases (CASPs) serve central roles are modulated by different factors, notably pro- and anti-apoptotic members of the BCL-2 family (68).

It has been reported that viruses have evolved different strategies to allow viral genomic products to inhibit apoptosis to counteract antiviral immunity (69). The viral genome-encoded 
Table I. Molecular targets of EBV-encoded miRs and their functions.

\begin{tabular}{|c|c|c|c|}
\hline EBV miR species & Molecular targets & Function of target molecules & (Refs.) \\
\hline miR-BART5 & PUMA & Pro-apoptotic effector of p53 & $(41)$ \\
\hline miR-BART3-5p & DICE1 & TSG in NPC & $(42)$ \\
\hline miR-BART9 & E-cadherin & $\begin{array}{l}\text { Promotes invasion and metastatic properties } \\
\text { of NPC cells }\end{array}$ & $(45)$ \\
\hline $\begin{array}{l}\text { miR-BART17-5p, miR-BART17-1-5p } \\
\text { and miR-BART17-16 }\end{array}$ & LMP1 & Oncogenic potential & $(19,49)$ \\
\hline miR-BART22 & LMP2 & Involvement in cellular transformation & $(19,50)$ \\
\hline
\end{tabular}

products include proteins and miRs to modulate host apoptosis. Whether BART miRs, which are frequently expressed in EBV-associated carcinomas, are required in the transformation of human epithelial cells remains unclear due to the lack of an experimental model for investigation. However, the data from the gastric carcinoma-derived cell line AGS support this speculation. Upon ectopic expression of BART miRs, the anchorage-independent growth of AGS cells, which are negative for the EBV genome but readily infected with EBV, was significantly enhanced and apoptosis was inhibited $(37,70,71)$. During the process, pro-apoptotic genes are downregulated by individual BART miRs $(38,39,53,72,73)$. A previous study reported that five of the 22 miR-BART pre-miRs were anti-apoptotic, as well as the seven identified pro-apoptotic cellular mRNA targets (six of which were novel) that significantly contributed to the observed anti-apoptotic phenotype (72).

An additional pro-apoptotic member of the BCL-2 family, the BH3-interacting domain death agonist protein (also known as BID), has been demonstrated to be downregulated in EBVaGC tissue samples and gastric carcinoma cell lines infected with EBV, resulting in inhibition of apoptosis (69). The expression of BAD, another BCL-2 family pro-apoptotic protein, has also been reported to be significantly lower in EBV-infected AGS-EBV cells when compared with that in EBV-negative AGS cells. In addition, five BART miRs that showed seed matching with the 3'-UTR of the BAD mRNA were identified. Of these identified miRs, only miR-BART20-5p reduced BAD expression when individually transfected into AGS cells. The EBV-encoded miRs were also demonstrated to reduce apoptosis and enhance cell growth $(70,71)$. miR-BART20-5p has been reported to increase chemoresistance to 5-fluorouracil and docetaxel $(53,73)$.

A total of $12 \mathrm{EBV}$ miRs were found to have one or more seed binding sites in the 3'-UTR of the caspase 3 (CASP3) mRNA, while nine induced significant repression of a full-length CASP3 reporter. Furthermore, three EBV miRs, including BART22, repressed the endogenous CASP3 protein (74). With regards to the crucial role of CASP3 in executing apoptosis, the data supports the notion that EBV-encoded miRs exert apoptosis inhibition to facilitate viral replication. It has also been reported that EBV-miR-BART15-3p sensitized GC cells to an anticancer drug, fluorouracil, through downregulation of Tax1-binding protein 1 (75).

EBV-encoded miRs target the cell cycle regulation. EBV-encoded miRs regulate diverse biological processes, including B-cell activation, cell proliferation, apoptosis and cell cycle entry through targeting multiple intracellular signaling pathways. Upregulated expression of PR domain zinc finger protein 1 (PRDM1), a master regulator of B-cell terminal differentiation and a tumor suppressor gene in aggressive lymphomas (such as in DLBCL), induced apoptosis and cell cycle arrest in the LCL. It has also been reported that PRDM1 is regulated by miR-BHRF1-2, which repressed the reporter activity by specific interactions with the seed region within the PRDM1 3'-UTR (76). The study also suggested that inhibition of miR-BHRF1-2 negatively regulated the cell cycle and decreases the expression of Small Cajal body-specific RNA 20, a small nucleolar RNA that is also downregulated by PRDM1 overexpression. The interaction between miR-BHRF1-2 and PRDM1 may thus be one of the mechanisms by which miR-BHRF1-2 promotes EBV lymphomagenesis (76).

When viral mutants with deletions of BHRF1 miRs infected target cells, the proliferation support of infected B cells was evidently lost early after infection. The cells with EBV lacking the BHRF1 miRs progressed through the cell cycle less efficiently and died due to apoptosis more frequently than cells infected with the parental EBV. According to phenotypic characterizations, EBV-encoded BHRF1 miRs supported B-cell activation mediated by EBV, but were not involved in maintaining viral latency $(74,76)$

The role of EBV-encoded miRs on the transformation of $B$ cells and growth of LCLs was examined using a mutant BHRF1 miR cluster. Deficient BHRF1 miRs still increased the number of B-cell-derived lymphoblastoid cell lines (LCLs) in the $\mathrm{S}$ phase, and this finding prompted a screening of the expression of cell cycle regulators. Reduced expression of the negative cell cycle regulator CDKN1B/p27 was identified after 30 days in culture, while CDKN1B/p27 expression was eliminated after 2 weeks of infection $(75,77)$. The reduced expression of p27 suggests that the effects of PTEN overexpression were counteracted in the infected cells. Indeed, 
PTEN blocks the repressive effects of Akt on p27 and thus increases p27 expression (76,78).

EBV miRs promote tumor progression. Certain EBV-encoded miRs are present in high levels in EBV-positive NPC tissues, as well as in the plasma of the patients. This raises the possibility that, similar to the viral proteins expressed, these miRs may contribute to the genesis and/or progression of NPC, and serve as an indicator for diagnosis and prognosis. Data have also suggested that EBV-encoded miRs promoted tumor metastasis (79).

The cellular tumor suppressor PTEN was reported to be directly targeted by EBV-miR-BART1 (43). The PTEN expression level has been shown to be reduced, while downstream PTEN-regulated signaling pathways are activated, including the PI3K-Akt, focal activating kinase-p130 and SHC-transforming protein 1-MAPK/ERK1/2 signaling pathways, and epithelial-mesenchymal transition is induced; hence, the migration, invasion and metastasis of the tumor cells is increased. Exogenous PTEN reversed all phenotypes generated by BART1, highlighting the role of PTEN in suppressing EBV-miR-BART-driven metastasis in NPC $(43,77)$. These data provided an insight into the EBV-mediated metastasis regulation and proposed novel clinical intervention strategies of NPC.

More than 20 BART miRs are detectable at abundant levels in EBV-positive NPC. Their expression profiles were studied in EBV latently infected Mutu I and Mutu III cell lines, EBV-positive NPC cells and noncancerous nasopharynx cells $(24,30)$. The results revealed that the miRs BART3, 7 and 13 were highly expressed and regularly secreted into the extracellular environment. High extracellular levels of these two EBV-BART-miRs were also identified in the plasma of patients with NPC, whereas the expression was absent in the plasma of non-NPC and healthy individual controls (79). However, the implication of miR-BART7 and 13 in the occurrence of NPC remains to be further elucidated.

miR-BART7 exists at significantly higher levels in patients with NPC when compared with those in healthy individuals, and this miR was detectable in all the patient plasma samples examined, independent of the EBV DNA level (78). Furthermore, in vitro expression of miR-BART7 enhanced the proliferation, migration and invasion of NPC cells, while NPC cells expressing miR-BART7 were found to be more resistant to cisplatin (35).

\section{EBV-encoded miRs as biomarkers for the diagnosis and prognosis of EBV-associated tumors}

EBV is associated with various types of non-Hodgkin's lymphoma, ranging from BL to opportunistic lymphomas, ARL and tumors that occur in immunologically compromised patients (80). Detection of genomic products of EBV and their antibodies in the peripheral blood is important for the diagnosis of these diseases. In hairy cell lymphoma, the effects of elevated organochlorines and antibodies against EBV proteins were determined (81). Titers of antibodies to the EBV early antigens and EBNAs were correlated to concentrations of organochlorines in order to evaluate the impact of these factors in the pathogenesis of hairy cell lymphoma (81).
The expression and their contribution of EBV miRs to the disease progression of chronic lymphocytic leukemia were further assessed in a previous study (80). It was reported that the expression level of EBV BHRF1-1, which is located immediately upstream and downstream of the BHRF1 open reading frame, and is frequently expressed in EBV latency III infections, was high in patients with chronic lymphocytic leukemia and was predictive of shorter overall survival. This predictive power was retained even when common prognostic factors were included in the multivariate analysis. The ability of BHRF1-1 expression levels to define the outcome in a validation group of patients was also confirmed (80).

miRs synthesized by different internal organs are released into the extracellular microenvironment in different forms, including exosomes, apoptotic bodies and microvesicles. They are tissue specific and detectable in the blood (82-84), and are stable in the serum or plasma with reproducible and consistent levels in different individuals of the same species $(83,84)$. Due to their persistence in circulation and their stable nature during diseases, miRs can serve as novel noninvasive diagnostic and prognostic biomarkers for diseases, including cancer $(84,85)$. The miR expression profile was examined in a variety of cancer types, and the expression signature of miRs can be used to distinguish cancer cells from normal cells (86) or one cancer type from another $(85,87-89)$, as well as to predict the response to a particular anticancer therapeutic agent (90) or the clinical outcome of patients (91-94).

miRs signatures are reported to be implicated in the prognosis of EBV-associated human tumors. For instance, NPC is very sensitive to radiation, and the majority of patients responded well to radiotherapy (95). Despite the efficacy of radiotherapy against this tumor, the problem of recurrence in cervical lymph nodes and distal metastasis is still encountered subsequent to treatment (96). Clinically, NPC has a high incidence of lymph node metastasis and occurs in a majority of the patients prior to their first visit to the clinic $(97,98)$. The demand to improve the efficacy of anti-NPC therapy has prompted the identification of genes that are implicated in lymph node involvement, which is an indicative of disease progression and therapeutic failure (99-101). Thus, the investigation of the role of miRs in NPC may provide a mechanistic explanation of lymph node metastasis, as well as more a sensitive indicator for the prognosis of NPC.

The discovery of molecular biomarkers that can be incorporated into the cancer staging system could improve the accuracy of prognostic prediction. Ideal biomarkers should be easily accessible and noninvasive; therefore, circulating nucleic acids are of great interest. Upregulation of EBV-encoded miRs (including BART1-3p, 2-5p, 5, 6-5p, 6-3p, 7, 8, 9, 14, 17-5p, 18-5p and 19-3p) was revealed in NPC biopsies using high throughput screening. It was observed that tumor and serum miR copy numbers from the patients and controls were correlated. The data further suggested that viral miRs are generally more upregulated than miRs of human origin and that their presence in the serum of patients with NPC was positively correlated with the copy numbers of the same miRs in tumor cells (23). Furthermore, cellular target genes were predicted by gene expression analysis using bioinformatics tools, revealing that the tumor suppressor PTEN and Wnt signaling pathways involved in NPC were targeted. High levels of the EBV-miRs 
BART2-5p, 6-5p and $17-5 p$ were revealed in the serum of patients with NPC, but not in that of healthy individuals. Their levels in NPC tumors were significantly correlated with the levels in serum samples, which may allow them to be used as diagnostic or prognostic markers (95).

Several EBV-encoded miRs, including BART1, 9, 16, 17 and 22, have been found to be expressed in NPC biopsies, while they were reported to have both cellular and viral targets, influencing in turn multiple host properties, such as growth, proliferation, survival and evasion of host immunity $(32,102,103)$. The biological function of serum-associated BART miRs remains unclear, although available evidence has suggested that its epigenetic regulation may target the host immune response $(65,66)$. Their expression and functional downregulation in circulating blood could enhance to attack virus-infected NPC cells by host immunity However, the downregulation of miRs expression in circulation and their function has been proved to enhance the attack of EBV infected NPC cells mediated by the host immunity (16). The BART miRs are possibly released into the blood from NPC cells in exosomes, apoptotic bodies or microvesicles.

The potential use of miR-BART3, 7 and 13 as NPC biomarkers has been evaluated, as they are highly expressed and regularly secreted into the extracellular environment of NPC cells (79). This study reported that the levels of miR-BART7 and miR-BART13, but not miR-BART3, were distinctly present in patients with NPC, with elevated levels being particularly apparent among patients in advanced stages of the disease. These data suggested that the EBV-encoded miR-BART7 and miR-BART13 may serve as novel serological biomarkers for the diagnosis of NPC and prediction of the efficacy of treatment.

The oncogenic potential of EBV-miR-BART7 has been suggested due to its high expression in NPC cells, as well as NPC biopsies $(16,21,30,33,34)$. It has been reported that in vitro expression of miR-BART7 enhanced the proliferation, migration and invasion of NPC cells, and enabled their high resistance to cisplatin (35). High-throughput gene expression analysis further suggested that EBV-miR-BART7 affects multiple cancer-related pathways $(33,79)$. A comparison was conducted between the circulating BART7 and EBV DNA (34). These data supported the usefulness of this viral $\mathrm{miR}$ as a biomarker of undifferentiated NPC, which is associated with EBV infection.

\section{Conclusions}

As epigenetic regulators of gene expression, miRs target numerous genes, accelerating their degradation to maintain EBV replication and persistence through inhibiting apoptosis and suppressing the host immune response. The effects significantly contribute to the achievement of malignancy of EBV-infected cells. Currently, it is accepted that EBV infection serves an important role in the early stages of NPC occurrence, and that it is implicated in the expansion of premalignant, dysplastic epithelial cell clones. In addition, LMP1 is regarded as a transforming protein that contributes to disease progression. Emerging evidence also supported the role of EBV-encoded miRs in the genesis of NPC. BART miRs are expressed in abundance in EBV-positive NPC, which may be due to their small size enabling them to escape innate immune defense. Finally, BART miRs are specific for EBV-infected NPC cells, and may therefore serve as good candidates for therapeutic targets of NPC. It is anticipated that a novel miR-based therapeutic strategy will be designed, either alone or in combination with pre-existing regimens, to treat EBV-infected malignancies.

\section{Acknowledgements}

The authors would like to thank Professor Ingemar Ernberg and Dr. Ljudmila Matskova (Karolinska Institutet, Sweden) for their stimulating discussions on the role of microRNAs in the regulation of gene expression.

\section{Funding}

The present study was supported by a research grant from the Medical Research Fund, Commission of Health and Family Plan of the Province of Guangdong (awarded to XZ; grant no. A2018356).

\section{Availability of data and materials}

Not applicable.

\section{Authors' contributions}

$\mathrm{XZ}, \mathrm{QQ}$ and ZH conceived, wrote and corrected the paper; $\mathrm{YY}$, MF and BZ wrote and revised the paper. All authors approved the final version of the manuscript before submission.

\section{Ethics approval and consent to participate}

Not applicable.

\section{Patient consent for publication}

Not applicable.

\section{Competing interests}

The authors declare that they have no competing interests.

\section{References}

1. Kutok JL and Wang F: Spectrum of Epstein Barr virus associated diseases. Annu Rev Pathol 1: 375-404, 2006.

2. Williams $\mathrm{H}$ and Crawford DH: Epstein-Barr virus: The impact of scientific advances on clinical practice. Blood 107: 862-869, 2006.

3. Lo KW, To KF and Huang DP: Focus on nasopharyngeal carcinoma. Cancer Cell 5: 423-428, 2004.

4. Cesarman E: Gammaherpesvirus and lymphoproliferative disorders in immunocompromised patients. Cancer Lett 305: 163-174, 2011.

5. Hsieh JJ and Hayward SD: Masking of the CBF1/RBPJ kappa transcriptional repression domain by Epstein-Barr virus EBNA2. Science 268: 560-563, 1995.

6. Yoshizaki T, Kondo S, Wakisaka N, Murono S, Endo K, Sugimoto H, Nakanishi S, Tsuji A and Ito M: Pathogenic role of Epstein-Barr virus latent membrane protein-1 in the development of nasopharyngeal carcinoma. Cancer Lett 337: 1-7, 2013.

7. Guasparri I, Bubman D and Cesarman E: EBV LMP2A affects LMP1-mediated NF-kappaB signaling and survival of lymphoma cells by regulating TRAF2 expression. Blood 111: 3813-3820, 2008. 
8. Marquitz AR, Mathur A, Edwards RH and Raab-Traub N: Host gene expression is regulated by two types of noncoding RNAs transcribed from the Epstein-Barr virus BamHI A rightward transcript region. J Virol 89: 11256-11268, 2015.

9. Bartel DP: MicroRNAs: Target recognition and regulatory functions. Cell 136: 215-233, 2009.

10. Winter J, Jung S, Keller S, Gregory RI and Diederichs S: Many roads to maturity: microRNA biogenesis pathways and their regulation. Nat Cell Biol 11: 228-234, 2009.

11. Han J, Lee Y, Yeom KH, Nam JW, Heo I, Rhee JK, Sohn SY, Cho Y, Zhang BT and Kim VN: Molecular basis for the recognition of primary microRNAs by the Drosha-DGCR8 complex. Cell 125: 887-901, 2006.

12. Busson P, Keryer C, Ooka T and Corbex M: EBV-associated nasopharyngeal carcinomas: From epidemiology to virus-targeting strategies. Trends Microbiol 12: 356-360, 2004.

13. Pfeffer S, Zavolan M, Grässer FA, Chien M, Russo JJ, Ju J, John B Enright AJ, Marks D, Sander C and Tuschl T: Identification of virus-encoded microRNAs. Science 304: 734-736, 2004.

14. Zhu JY, Pfuhl T, Motsch N, Barth S, Nicholls J, Grässer F and Meister G: Identification of novel Epstein-Barr virus microRNA genes from nasopharyngeal carcinomas. J Virol 83: 3333-3341, 2009 .

15. Edwards RH, Marquitz AR and Raab-Traub N: Epstein-Barr virus BART microRNAs are produced from a large intron prior to splicing. J Virol 82: 9094-9106, 2008.

16. Qiu J, Cosmopoulos K, Pegtel M, Hopmans E, Murray P, Middeldorp J, Shapiro M and Thorley-Lawson DA: A novel persistence associated EBV miRNA expression profile is disrupted in neoplasia. PLoS Pathog 7: e1002193, 2011.

17. Tsai CY, Liu YY, Liu KH, Hsu JT, Chen TC, Chiu CT and Yeh TS: Comprehensive profiling of virus microRNAs of Epstein-Barr virus-associated gastric carcinoma: Highlighting the interactions of ebv-Bart9 and host tumor cells. J Gastroenterol Hepatol 32: 82-91, 2017.

18. Sakamoto K, Sekizuka T, Uehara T, Hishima T, Mine S, Fukumoto H, Sato Y, Hasegawa H, Kuroda M and Katano H: Next-generation sequencing of miRNAs in clinical samples of Epstein-Barr virus-associated B-cell lymphomas. Cancer Med 6: 605-618, 2017

19. Lo AKF, Dawson CW, Jin DY and Lo KW: The pathological roles of BART miRNAs in nasopharyngeal carcinoma. J Pathol 227: 392-403, 2012.

20. Baer R, Bankier AT, Biggin MD, Deininger PL, Farrell PJ, Gibson TJ, Hatfull G, Hudson GS, Satchwell SC, Séguin C, et al: DNA sequence and expression of the B95-8 Epstein-Barr virus genome. Nature 310: 207-211, 1984.

21. Pratt ZL, Kuzembayeva M, Sengupta S and Sugden B: The microRNAs of Epstein-Barr Virus are expressed at dramatically differing levels among cell lines. Virology 386: 387-397, 2009

22. Xia T, O'Hara A, Araujo I, Barreto J, Carvalho E, Sapucaia JB Ramos JC, Luz E, Pedroso C, Manrique M, et al: EBV microRNAs in primary lymphomas and targeting of CXCL-11 by ebv-mir-BHRF1-3. Cancer Res 68: 1436-1442, 2008

23. Kim DN, Chae HS, Oh ST, Kang JH, Park CH, Park WS, Takada K, Lee JM, Lee WK and Lee SK: Expression of viral microRNAs in Epstein-Barr virus-associated gastric carcinoma. J Virol 81: 1033-1036, 2007.

24. Cosmopoulos K, Pegtel M, Hawkins J, Moffett H, Novina C, Middeldorp J and Thorley-Lawson DA: Comprehensive profiling of Epstein-Barr virus microRNAs in nasopharyngeal carcinoma. J Virol 83: 2357-2367, 2009.

25. Chen SJ, Chen GH, Chen YH, Liu CY, Chang KP, Chang YS and Chen HC: Characterization of Epstein-Barr virus miRNAome in nasopharyngeal carcinoma by deep sequencing. PLoS One 5: pii: e12745, 2010

26. Motsch N, Alles J, Imig J, Zhu J, Barth S, Reineke T, Tinguely M, Cogliatti S, Dueck A, Meister G, et al: MicroRNA profiling of Epstein-Barr virus-associated NK/T-cell lymphomas by deep sequencing. PLoS One 7: e42193, 2012.

27. Jun SM, Hong YS, Seo JS, Ko YH, Yang CW and Lee SK: Viral microRNA profile in Epstein-Barr virus-associated peripheral T cell lymphoma. Brit J Haematol 142: 320-323, 2008

28. Arikawa J, Tokunaga M, Tashiro Y, Tanaka S, Sato E, Haraguchi K, Yamamoto A, Toyohira O and Tsuchimochi A: Epstein-Barr virus-positive multiple early gastric cancers and dysplastic lesions: A case report. Pathol Int 47: 730-734, 1997.

29. Fukayama M: Epstein-Barr virus and gastric carcinoma. Pathol Int 60: $337-350,2010$
30. Wong AM, Kong KL, Tsang JW, Kwong DL and Guan XY: Profiling of Epstein-Barr virus-encoded microRNAs in nasopharyngeal carcinoma reveals potential biomarkers and oncomirs. Cancer 118: 698-710, 2012.

31. Chen C, Ridzon DA, Broomer AJ, Zhou Z, Lee DH, Nguyen JT, Barbisin M, Xu NL, Mahuvakar VR, Andersen MR, et al: Real-time quantification of microRNAs by stem-loop RT-PCR Nucleic Acids Res 33: e179, 2005.

32. Amoroso R, Fitzsimmons L, Thomas WA, Kelly GL, Rowe M and Bell AI: Quantitative studies of Epstein-Barr virus-encoded microRNAs provide novel insights into their regulation. J Virol 85: 996-1010, 2011.

33. Chan JY, Gao W, Ho WK, Wei WI and Wong TS: Overexpression of Epstein-Barr virus-encoded microRNA-BART7 in undifferentiated nasopharyngeal carcinoma. Anticancer Res 32: 3201-3210, 2012.

34. Cai X, Schäfer A, Lu S, Bilello JP, Desrosiers RC, Edwards R, Raab-Traub N and Cullen BR: Epstein-Barr virus microRNAs are evolutionarily conserved and differentially expressed. PLoS Pathog 2: e23, 2006.

35. Neuhierl B and Delecluse HJ: Molecular genetics of DNA viruses: Recombinant virus technology. Methods Mol Biol 292: 353-370, 2005

36. Feederle R, Linnstaedt SD, Bannert H, Lips H, Bencun M, Cullen BR and Delecluse HJ: A viral microRNA cluster strongly potentiates the transforming properties of a human herpesvirus. PLoS Pathog 7: e1001294, 2011.

37. Tsao SW, Tsang CM and Lo KW: Epstein-Barr virus infection and nasopharyngeal carcinoma. Philos Trans R Soc Lond B Biol Sci 372: pii: 20160270, 2017.

38. Young LS and Dawson CW: Epstein-Barr virus and nasopharyngeal carcinoma. Chin J Cancer 33: 581-590, 2014

39. Zheng X, Wang J, Wei L, Peng Q, Gao Y, Fu Y, Lu Y, Qin Z, Zhang X, Lu J, et al: Epstein-Barr virus MicroRNA miR-BART5-3p inhibits p53 expression. J Virol 92: pii: e01022-18, 2018.

40. Marquitz AR, Mathur A, Nam CS and Raab-Traub N: The Epstein-Barr virus BART microRNAs target the pro-apoptotic protein Bim. Virology 412: 392-400, 2011.

41. Choy EY, Siu KL, Kok KH, Lung RW, Tsang CM, To KF, Kwong DL, Tsao SW and Jin DY: An Epstein-Barr virus-encoded microRNA targets PUMA to promote host cell survival. J Exp Med 205: 2551-2560, 2008

42. Lei T, Yuen KS, Xu R, Tsao SW, Chen H, Li M, Kok KH and Jin DY: Targeting of DICE1 tumor suppressor by Epstein-Barr virus-encoded miR-BART3* microRNA in nasopharyngeal carcinoma. Int J Cancer 133: 79-87, 2013.

43. Cai L, Ye Y, Jiang Q, Chen Y, Lyu X, Li J, Wang S, Liu T, Cai H, Yao K, et al: Epstein-Barr virus-encoded microRNA BART1 induces tumour metastasis by regulating PTEN-dependent pathways in nasopharyngeal carcinoma. Nat Commun 6: 7353 , 2015.

44. Cai LM, Lyu XM, Luo WR, Cui XF, Ye YF, Yuan CC, Peng QX, Wu DH, Liu TF, Wang E, et al: EBV-miR-BART7-3p promotes the EMT and metastasis of nasopharyngeal carcinoma cells by suppressing the tumor suppressor PTEN. Oncogene 34: 2156-2166, 2015 .

45. Hsu CY, Yi YH, Chang KP, Chang YS, Chen SJ and Chen HC: The Epstein-Barr virus-encoded microRNA MiR-BART9 promotes tumor metastasis by targeting E-cadherin in nasopharyngeal carcinoma. PLoS Pathog 10: e1003974, 2014.

46. Dölken L, Malterer G, Erhard F, Kothe S, Friedel CC, Suffert G, Marcinowski L, Motsch N, Barth S, Beitzinger M, et al: Systematic analysis of viral and cellular microRNA targets in cells latently infected with human gamma-herpesviruses by RISC immunoprecipitation assay. Cell Host Microbe 7: 324-334, 2010.

47. Iizasa H, Wulff BE, Alla NR, Maragkakis M, Megraw M, Hatzigeorgiou A, Iwakiri D, Takada K, Wiedmer A, Showe L, et al: Editing of Epstein-Barr virus-encoded BART6 microRNAs controls their dicer targeting and consequently affects viral latency. J Biol Chem 285: 33358-33370, 2010.

48. Nachmani D, Stern-Ginossar N, Sarid R and Mandelboim O: Diverse herpesvirus microRNAs target the stress-induced immune ligand MICB to escape recognition by natural killer cells. Cell Host Microbe 5: 376-385, 2009.

49. Lo AK, To KF, Lo KW, Lung RW, Hui JW, Liao G and Hayward SD: Modulation of LMP1 protein expression by EBV-encoded microRNAs. Proc Natl Acad Sci USA 104: 16164-16169, 2007 
50. Lung RW, Tong JH, Sung YM, Leung PS, Ng DC, Chau SL, Chan AW, Ng EK, Lo KW and To KF: Modulation of LMP2A expression by a newly identified Epstein-Barr virus-encoded microRNA miR-BART22. Neoplasia 11: 1174-1184, 2009.

51. Zhang X, Dawson CW, He Z and Huang P: Immune evasion strategies of the human gamma-herpesviruses: Implications for viral tumorigenesis. J Med Virol 84: 272-281, 2012.

52. Seto E, Moosmann A, Gromminger S, Walz N, Grundhoff A and Hammerschmidt W: Micro RNAs of Epstein-Barr virus promote cell cycle progression and prevent apoptosis of primary human B cells. PLoS Pathog 6: e1001063, 2010.

53. Vereide DT, Seto E, Chiu YF, Hayes M, Tagawa T, Grundhoff A, Hammerschmidt W and Sugden B: Epstein-Barr virus maintains lymphomas via its miRNAs. Oncogene 33: 1258-1264, 2014

54. Ramalingam D, Kieffer-Kwon P and Ziegelbauer JM: Emerging themes from EBV and KSHV microRNA targets. Viruses 4 1687-1710, 2012.

55. Fernandes Q, Merhi M, Raza A, Inchakalody VP, Abdelouahab N, Zar Gul AR, Uddin S and Dermime S: Role of Epstein-Barr virus in the pathogenesis of head and neck cancers and its potential as an immunotherapeutic target. Front in Oncol 8: 257, 2018.

56. Albanese M, Tagawa T, Buschle A and Hammerschmidt W: MicroRNAs of Epstein-Barr virus control innate and adaptive antiviral immunity. J Virol 91: pii: e01667-16, 2017.

57. Poling BC, Price AM, Luftig MA and Cullen BR: The Epstein-Barr virus miR-BHRF1 microRNAs regulate viral gene expression in cis. Virology 512: 113-123, 2017.

58. Kim Y, Lee S, Kim S, Kim D, Ahn JH and Ahn K: Human cytomegalovirus clinical strain-specific microRNA miR-UL148D targets the human chemokine RANTES during infection. PLoS Pathog 8: e1002577, 2012.

59. Haneklaus M, Gerlic M, Kurowska-Stolarska M, Rainey AA, Pich D, McInnes IB, Hammerschmidt W, O'Neill LA and Masters SL: Cutting edge: miR-223 and EBV miR-BART15 regulate the NLRP3 inflammasome and IL-1 $\beta$ production. J Immunol 189: 3795-3799, 2012.

60. Kim S, Lee S, Shin J, Kim Y, Evnouchidou I, Kim D, Kim YK, Kim YE, Ahn JH, Riddell SR, et al: Human cytomegalovirus microRNA miR-US4-1 inhibits CD8(+) T cell responses by targeting the aminopeptidase ERAP1. Nat Immunol 12: 984-991, 2011.

61. Bauman Y, Nachmani D, Vitenshtein A, Tsukerman P, Drayman N, Stern-Ginossar N, Lankry D, Gruda R and Mandelboim O: An identical miRNA of the human JC and BK polyoma viruses targets the stress-induced ligand ULBP3 to escape immune elimination. Cell Host Microbe 9: 93-102, 2011.

62. Abend JR, Ramalingam D, Kieffer-Kwon P, Uldrick TS, Yarchoan R and Ziegelbauer JM: Kaposi's sarcoma-associated herpesvirus microRNAs target IRAK1 and MYD88, two components of the toll-like receptor/interleukin-1R signaling cascade, to reduce inflammatory-cytokine expression. J Virol 86 11663-11674, 2012

63. Lei X, Bai Z, Ye F, Xie J, Kim CG, Huang Y and Gao SJ Regulation of NF-kappaB inhibitor IkappaBalpha and viral replication by a KSHV microRNA. Nat Cell Biol 12: 193-199, 2010.

64. Liang D, Gao Y, Lin X, He Z, Zhao Q, Deng Q and Lan K: A human herpesvirus miRNA attenuates interferon signaling and contributes to maintenance of viral latency by targeting IKKe. Cell Res 21: 793-806, 2011

65. Hooykaas MJG, van Gent M, Soppe JA, Kruse E, Boer IGJ, van Leenen D, Groot Koerkamp MJA, Holstege FCP, Ressing ME, Wiertz EJHJ and Lebbink RJ: EBV MicroRNA BART16 suppresses type I IFN signaling. J Immunol 198: 4062-4073, 2017.

66. Skinner CM, Ivanov NS, Barr SA, Chen Y and Skalsky RL: An Epstein-Barr virus microrna blocks interleukin-1 (IL-1) signaling by targeting IL-1 receptor 1. J Virol 91: pii: e00530-17, 2017.

67. Dillon CP and Green DR: Molecular cell biology of apoptosis and necroptosis in cancer. Adv Exp Med Biol 930: 1-23, 2016.

68. Kvansakul M, Caria S and Hinds MG: The Bcl-2 family in host-virus interactions. Viruses 9: pii: E290, 2017.

69. Kang D, Skalsky RL and Cullen BR: EBV BART microRNAs target multiple pro-apoptotic cellular genes to promote epithelial cell survival. PLoS Pathog 11: e1004979, 2015.

70. Kim H, Choi H and Lee SK: Epstein-Barr virus miR-BART20-5p regulates cell proliferation and apoptosis by targeting BAD. Cancer Lett 356: 733-742, 2015.
71. Kim H, Choi $\mathrm{H}$ and Lee SK: Epstein-Barr virus MicroRNA miR-BART20-5p suppresses lytic induction by inhibiting BAD-mediated caspase-3-dependent apoptosis. J Virol 90: 1359-1368, 2015

72. Marquitz AR, Mathur A, Shair KH and Raab-Traub N: Infection of Epstein-Barr virus in a gastric carcinoma cell line induces anchorage independence and global changes in gene expression. Proc Natl Acad Sci USA 109: 9593-9598, 2012.

73. Shinozaki-Ushiku A, Kunita A, Isogai M, Hibiya T, Ushiku T, Takada K and Fukayama M: Profiling of virus-encoded microRNAs in Epstein-Barr virus-associated gastric carcinoma and their roles in gastric carcinogenesis. J Virol 89: 5581-5591, 2015.

74. Harold C, Cox D and Riley KJ: Epstein-Barr viral microRNAs target caspase 3. Virol J 13: 145, 2016.

75. Choi $\mathrm{H}$ and Lee SK: TAX1BP1 downregulation by EBV-miR-BART15-3p enhances chemosensitivity of gastric cancer cells to 5-FU. Arch Virol 162: 369-377, 2017.

76. Ma J, Nie K, Redmond D, Liu Y, Elemento O, Knowles DM and Tam W: EBV-miR-BHRF1-2 targets PRDM1/Blimp1: Potential role in EBV lymphomagenesis. Leukemia 30: 594-604, 2016.

77. Bernhardt K, Haar J, Tsai MH, Poirey R, Feederle R and Delecluse HJ: A viral microRNA cluster regulates the expression of PTEN, p27 and of a bcl-2 homolog. PLoS Pathog 12: e1005405, 2016.

78. Engelman JA, Luo J and Cantley LC: The evolution of phosphatidylinositol 3-kinases as regulators of growth and metabolism. Nat Rev Genet 7: 606-619, 2006.

79. Zhang G, Zong J, Lin S, Verhoeven RJ, Tong S, Chen Y, Ji M, Cheng W, Tsao SW, Lung M, et al: Circulating Epstein-Barr virus microRNAs miR-BART7 and miR-BART13 as biomarkers for nasopharyngeal carcinoma diagnosis and treatment. Int J Cancer 136: E301-E312, 2015.

80. Ferrajoli A, Ivan C, Ciccone M, Shimizu M, Kita Y, Ohtsuka M, D'Abundo L, Qiang J, Lerner S, Nouraee N, et al: Epstein-Barr virus microRNAs are expressed in patients with chronic lymphocytic leukemia and correlate with overall survival. EBioMedicine 2: 572-582, 2015.

81. Nordström M, Hardell L, Lindström G, Wingfors H, Hardell K and Linde A: Concentrations of organochlorines related to titers to Epstein-Barr virus early antigen IgG as risk factors for hairy cell leukemia. Environ Health Perspect 108: 441-445, 2000.

82. Sempere LF: Tissue slide-based microRNA characterization of tumors: How detailed could diagnosis become for cancer medicine? Expert Rev Mol Diagn 14: 853-869, 2014.

83. Mishra PJ: MicroRNAs as promising biomarkers in cancer diagnostics. Biomark Res 2: 19, 2014.

84. Ohshima K, Inoue K, Fujiwara A, Hatakeyama K, Kanto K, Watanabe Y, Muramatsu K, Fukuda Y, Ogura S, Yamaguchi K and Mochizuki T: Let-7 microRNA family is selectively secreted into the extracellular environment via exosomes in a metastatic gastric cancer cell line. PLoS One 5: e13247, 2010.

85. Zhao H, Shen J, Medico L, Wang D, Ambrosone CB and Liu S: A pilot study of circulating miRNAs as potential biomarkers of early stage breast cancer. PLoS One 5: e13735, 2010.

86. Pigati L, Yaddanapudi SC, Iyengar R, Kim DJ, Hearn SA, Danforth D, Hastings ML and Duelli DM: Selective release of microRNA species from normal and malignant mammary epithelial cells. PLoS One 5: e13515, 2010.

87. Wei R, Huang GL, Zhang MY, Li BK, Zhang HZ, Shi M, Chen XQ, Huang L, Zhou QM, Jia WH, et al: Clinical significance and prognostic value of microRNA expression signatures in hepatocellular carcinoma. Clin Cancer Res 19: 4780-4791, 2013.

88. Cheng H, Zhang L, Cogdell DE, Zheng H, Schetter AJ, Nykter M, Harris CC, Chen K, Hamilton SR and Zhang W: Circulating plasma MiR-141 is a novel biomarker for metastatic colon cancer and predicts poor prognosis. PLoS One 6: e17745, 2011

89. Subramanian S, Lui WO, Lee CH, Espinosa I, Nielsen TO, Heinrich MC, Corless CL, Fire AZ and van de Rijn M: MicroRNA expression signature of human sarcomas. Oncogene 27: 2015-2026, 2008

90. Osaki M, Takeshita F and Ochiya T: MicroRNAs as biomarkers and therapeutic drugs in human cancer. Biomarkers 13: 658-670, 2008.

91. Nair VS, Maeda LS and Ioannidis JP: Clinical outcome prediction by microRNAs in human cancer: A systematic review. J Natl Cancer Inst 104: 528-540, 2012. 
92.Hu Z, Chen X, Zhao Y, Tian T, Jin G, Shu Y, Chen Y, Xu L, Zen $K$, Zhang $C$ and Shen H: Serum microRNA signatures identified in a genome-wide serum microRNA expression profiling predict survival of non-small-cell lung cancer. J Clin Oncol 28: 1721-1726, 2010.

93. Zuo Z, Calin GA, de Paula HM, Medeiros LJ, Fernandez MH, Shimizu M, Garcia-Manero G and Bueso-Ramos CE: Circulating microRNAs let-7a and miR-16 predict progression-free survival and overall survival in patients with myelodysplastic syndrome. Blood 118: 413-415, 2011.

94. Esquela-Kerscher A and Slack FJ: Oncomirs-microRNAs with a role in cancer. Nat Rev Cancer 6: 259-269, 2006.

95. Zhang MX, Li J, Shen GP, Zou X, Xu JJ, Jiang R, You R, Hua YJ, Sun Y, Ma J, et al: Intensity-modulated radiotherapy prolongs the survival of patients with nasopharyngeal carcinoma compared with conventional two-dimensional radiotherapy: A 10-year experience with a large cohort and long follow-up. Eur J Cancer 51: 2587-2595, 2015

96. Chung IC, Chen LC, Chung AK, Chao M, Huang HY, Hsueh C, Tsang NM, Chang KP, Liang Y, Li HP and Chang YS: Matrix metalloproteinase 12 is induced by heterogeneous nuclear ribonucleoprotein $\mathrm{K}$ and promotes migration and invasion in nasopharyngeal carcinoma. BMC Cancer 14: 348, 2014.

97. Yi W, Li X, Liu Z, Jiang C, Niu D and Xia Y: A risk score model for the metastasis of level Ib lymph node based on the clinicopathological features of nasopharyngeal carcinoma in a large sample. Mol Clin Oncol 2: 789-797, 2014.
98. Shi Q, Shen C, Kong L, Wang X, Ding J, Gao Y, Xu T and Hu C Involvement of both cervical lymph nodes and retropharyngeal lymph nodes has prognostic value for $\mathrm{N} 1$ patients with nasopharyngeal carcinoma. Radiat Oncol 9: 7, 2014.

99. Wang HZ, Cao CN, Luo JW, Yi JL, Huang XD, Zhang SP, Wang K, Qu Y, Xiao JP, Li SY, et al: High-risk factors of parotid lymph node metastasis in nasopharyngeal carcinoma: A case-control study. Radiat Oncol 11: 113, 2016.

100. Tang LL, Tang XR, Li WF, Chen L, Tian L, Lin AH, Sun Y and Ma J: The feasibility of contralateral lower neck sparing intensity modulation radiated therapy for nasopharyngeal carcinoma patients with unilateral cervical lymph node involvement. Oral Oncol 69: 68-73, 2017.

101. Zhang Y, Li WF, Chen L, Mao YP, Guo R, Zhang F, Peng H, Liu LZ, Li L, Liu Q and Ma J: Prognostic value of parotid lymph node metastasis in patients with nasopharyngeal carcinoma receiving intensity-modulated radiotherapy. Sci Rep 5: 13919, 2015.

102. Tagawa T, Albanese M, Bouvet M, Moosman A, Mautner J, Heissmeyer V Zielinski C, Hoser J, Hastreiter M, Hayes M, et al: Epstein-Barr viral miRNAs inhibit antiviral CD4+ T cell responses targeting IL-12 and peptide processing. J Exp Med 213: 2065-2080, 2016.

103. Feederle R, Haar J, Bernhardt K, Linnstaedt SD, Bannert H, Lips H, Cullen BR and Delecluse HJ: The members of an Epstein-Barr virus microRNA cluster cooperate to transform B lymphocytes. J Virol 85: 9801-9810, 2011. 